\title{
Gakushuin University (Tokyo)
}

\begin{tabular}{|c|c|c|c|c|}
\hline $\begin{array}{c}\text { Laboratory } \\
\text { number }\end{array}$ & $\begin{array}{l}\text { Published } \\
\text { reference }\end{array}$ & $\begin{array}{l}\text { Original da:e or } \\
\quad \text { other value }\end{array}$ & $\begin{array}{l}\text { Corrected date } \\
\text { or other value }\end{array}$ & $\partial \mathrm{C}^{14}, \%$ \\
\hline GaK-51 & $83: 91$ & $630 \pm 110$ & & \\
\hline-52 & $83: 91$ & $2190 \pm 130$ & & \\
\hline-53 & $83: 91$ & $2410 \pm 140$ & & \\
\hline-54 & $83: 92$ & $1600 \pm 150$ & & \\
\hline-55 & $83: 91$ & $1730 \pm 70$ & & \\
\hline-61 & $83: 93$ & $240 \pm 70$ & & \\
\hline-62 & $83: 93$ & $360 \pm 45$ & & \\
\hline-63 & $83: 93$ & $290 \pm 70$ & & \\
\hline-64 & $83: 93$ & $380 \pm 60$ & & \\
\hline-65 & $83: 93$ & $380 \pm 60$ & & \\
\hline-66 & $83: 93$ & $780 \pm 100$ & & \\
\hline-67 & $83: 93$ & $1210 \pm 80$ & & \\
\hline-68 & $83: 93$ & $890 \pm 65$ & & \\
\hline-70 & $83: 93$ & $1060 \pm 50$ & & \\
\hline-71 & $83: 93$ & $670 \pm 50$ & & \\
\hline-72 & $83: 94$ & $910 \pm 70$ & & \\
\hline-73 & $83: 94$ & $810 \pm 100$ & & \\
\hline-74 & $83: 93$ & $1410 \pm 60$ & & \\
\hline .75 & $83: 94$ & $1555 \pm 90$ & & \\
\hline-76 & $83: 94$ & $1435 \pm 55$ & & \\
\hline-77 & $83: 94$ & $1460 \pm 70$ & & \\
\hline-78 & $83: 94$ & $1410 \pm 90$ & & \\
\hline .79 & $83: 94$ & $1015 \pm 85$ & & \\
\hline-106 & $83: 91$ & $2680 \pm 150$ & & \\
\hline .107 & $83: 91$ & $3580 \pm 130$ & & \\
\hline-108 & $83: 91$ & $540 \pm 70$ & & \\
\hline .109 & $83: 92$ & $3030 \pm 70$ & & \\
\hline .110 & $83: 84$ & $9350 \pm 190$ & & \\
\hline-111 & $83: 85$ & $19,800 \pm 600$ & & \\
\hline-112 & $83: 85$ & $26,000 \pm 800$ & & \\
\hline-113 & $83: 86$ & $>30,000$ & & \\
\hline-114 & $83: 92$ & $1610 \pm 90$ & & \\
\hline-115 & $83: 86$ & $3180 \pm 180$ & & \\
\hline-117 & $83: 90$ & $730 \pm 90$ & & \\
\hline-118 & $83: 89$ & $>30,000$ & & \\
\hline .119 & $83: 87$ & $520 \pm 80$ & & \\
\hline-120 & $83: 88$ & $>30,000$ & & \\
\hline-121 & $83: 89$ & $22,200 \pm 900$ & & \\
\hline-122 & $83: 90$ & $13,900 \pm 330$ & & \\
\hline-123 & $83: 89$ & $>30,000$ & & \\
\hline-124 & $83: 89$ & $23,000 \pm 600$ & & \\
\hline-125 & $83: 89$ & $21,450 \pm 800$ & & \\
\hline-126 & $83: 88$ & $>30,000$ & & \\
\hline-129 & $83: 89$ & $8330 \pm 140$ & & \\
\hline-130 & $83: 90$ & $110 \pm 80$ & & \\
\hline-131 & $83: 87$ & $>30,000$ & & \\
\hline-132 & $83: 90$ & $1090 \pm 60$ & & \\
\hline-133 & $83: 90$ & $310 \pm 80$ & & \\
\hline-134 & $83: 87$ & $2470 \pm 70$ & & \\
\hline .135 & $83: 87$ & $2500 \pm 70$ & & \\
\hline-136 & $83: 87$ & $940 \pm 60$ & & \\
\hline-137 & $83: 88$ & $980 \pm 200$ & & \\
\hline-138 & $83: 88$ & $9870 \pm 290$ & & \\
\hline-139 & $83: 88$ & $9310 \pm 200$ & & \\
\hline-140 & $83: 88$ & $17,710 \pm 440$ & & \\
\hline-143 & $83: 88$ & $>30,000$ & & \\
\hline .144 & $83: 88$ & $12,300 \pm 230$ & & \\
\hline-145 & $83: 88$ & $11,500 \pm 230$ & & \\
\hline-146 & $83: 88$ & $11,740 \pm 200$ & & \\
\hline
\end{tabular}

A.D./ B.C. dat $\epsilon$ A.D. 1320 240 B.C. 460 B.C.

A.D. 350 A.D. 220

A.D. 710

A.D. 1590

A.D. 1660

A.D. 1570

A.D. 1570

A.D. 1170

A.D. 740

A.D. 1060

A.D. 890

A.D. 1280

A.D. 1040

A.D. 1140

A.D. 540

A.D. 395

A.D. 515

A.D. 490

A.D. 540

A.D. 935

730 B.C.

1630 B.C.

A.D. 1410

1080 B.C.

7400 B.C.

17,850 B.C.

24,050 B.C.

A.D. 340

1230 B.C.

A.D. 1220

A.D. 1430

20,250 B.C.

11,950 B.C.

21,050 B.C.

19,500 B.C.

6380 B.C.

A.D. 1840

A.D. 860

A.D. 1640

520 B.C.

550 B.C.

A.D. 1010

A.D. 970

7920 B.C.

7360 B.C.

15,760 B.C.

10,350 B.C.

9550 B.C.

9790 B.C. 


\begin{tabular}{|c|c|c|c|c|}
\hline $\begin{array}{c}\text { Laboratory } \\
\text { number }\end{array}$ & $\begin{array}{c}\text { Published } \\
\text { reference }\end{array}$ & $\begin{array}{l}\text { Original date or } \\
\quad \text { other value }\end{array}$ & $\begin{array}{l}\text { Corrected date } \\
\text { or other value }\end{array}$ & $\partial \mathrm{C}^{14}, \%$ \\
\hline-147 & $83: 88$ & $15,660 \pm 740$ & & \\
\hline-152 & $83: 92$ & $590 \pm 80$ & & \\
\hline-153 & $83: 92$ & $610 \pm 80$ & & \\
\hline-154 & $83: 92$ & $660 \pm 80$ & & \\
\hline-155 & $83: 92$ & $480 \pm 70$ & & \\
\hline-158 & $83: 86$ & $>30,000$ & & \\
\hline-159 & $83: 89$ & $13,130 \pm 230$ & & \\
\hline-160 & $83: 93$ & $15,800 \pm 140$ & & \\
\hline-161 & $83: 86$ & $15,750 \pm 390$ & & \\
\hline$-163 \mathrm{a}^{1}$ & $83: 86$ & $3380 \pm 160$ & & \\
\hline$-163 b^{1}$ & $83: 85$ & $>30,000$ & & \\
\hline-164 & $83: 85$ & $>30,000$ & & \\
\hline-165 & $83: 85$ & $>30,000$ & & \\
\hline-166 & $83: 85$ & $4840 \pm 120$ & & \\
\hline-167 & $83: 85$ & $12,730 \pm 340$ & & \\
\hline-168 & $83: 86$ & $4260 \pm 110$ & & \\
\hline-169 & $83: 86$ & $2820 \pm 90$ & & \\
\hline-170 & $83: 92$ & $3060 \pm 110$ & & \\
\hline-171 & $83: 88$ & $11,330 \pm 260$ & & \\
\hline-172 & $83: 88$ & $11,840 \pm 290$ & & \\
\hline-175 & $137: 200$ & $28,500 \pm 2500$ & & \\
\hline-176 & $137: 201$ & $9070 \pm 400$ & & \\
\hline-177 & $137: 202$ & $>30,000$ & & \\
\hline-178 & $137: 202$ & $2490 \pm 100$ & & \\
\hline-179 & $110: 113$ & $>30,000$ & & \\
\hline-180 & $110: 113$ & $17,400 \pm 900$ & & \\
\hline-181 & $110: 113$ & $8930 \pm 230$ & & \\
\hline-182 & $110: 113$ & $9450 \pm 350$ & & \\
\hline-184 & $110: 113$ & $10,400 \pm 400$ & & \\
\hline-186 & $110: 116$ & $1610 \pm 110$ & & \\
\hline-187 & $110: 116$ & $1070 \pm 80$ & & \\
\hline-188 & $110: 116$ & $4150 \pm 400$ & & \\
\hline-189 & $110: 117$ & $990 \pm 140$ & & \\
\hline-190 & $110: 117$ & $1420 \pm 70$ & & \\
\hline-191 & $110: 117$ & $1310 \pm 120$ & & \\
\hline-192 & $110: 196$ & $1990 \pm 110$ & & \\
\hline-193 & $110: 116$ & $1850 \pm 90$ & & \\
\hline-194 & $110: 116$ & $3530 \pm 120$ & & \\
\hline-195 & $110: 116$ & $1750 \pm 100$ & & \\
\hline-197 & $110: 115$ & $5870 \pm 160$ & & \\
\hline-198 & $110: 115$ & $5440 \pm 120$ & & \\
\hline-200 & $83: 90$ & $>30,000$ & & \\
\hline-201 & $83: 90$ & $3840 \pm 110$ & & \\
\hline-203 & $110: 111$ & $3800 \pm 130$ & & \\
\hline$-204 b$ & $110: 111$ & $27,800 \pm 2000$ & & \\
\hline-205 & $110: 114$ & $12,700 \pm 260$ & & \\
\hline-206 & $110: 110$ & $24,200 \pm 1100$ & & \\
\hline-207 & $110: 111$ & $24,950 \pm 1250$ & & \\
\hline-208 & $110: 113$ & $6370 \pm 110$ & & \\
\hline-209 & $110: 114$ & $25,400 \pm 1150$ & & \\
\hline-210 & $110: 117$ & $14,800 \pm 350$ & & \\
\hline-211 & $110: 109$ & $22,000 \pm 850$ & & \\
\hline-212 & $110: 117$ & $15,800 \pm 380$ & & \\
\hline-212 & $151: 21$ & $180 \pm 60$ & & \\
\hline-213 & $110: 110$ & $2270 \pm 100$ & & \\
\hline-215 & $151: 21$ & modern & & \\
\hline-216 & $151: 21$ & modern & & \\
\hline-217 & $151: 21$ & $160 \pm 90$ & & \\
\hline-218 & $151: 21$ & $940 \pm 90$ & & \\
\hline-219 & $110: 114$ & $8060 \pm 500$ & & \\
\hline-220 & $110: 114$ & $24,300 \pm 1150$ & & \\
\hline
\end{tabular}

A.D./

B.C. date

13,710 B.C.

A.D. 1360

A.D. 1340

A.D. 1290

A.D. 1470

11,180 B.C.

13,850 B.C.

13,800 B.C.

1430 B.C.

2890 B.C.

10,780 B.C.

2310 B.C.

870 B.C.

1110 B.C.

9380 B.C.

9890 B.C.

26,550 B.C.

7120 B.C.

540 B.C.

15,450 B.C.

6980 B.C.

7500 B.C.

8450 B.C.

A.D. 340

A.D. 880

2200 B.C.

A.D. 960

A.D. 960

A.D. 640

40 B.C.

A.D. 100

1580 B.C.

A.D. 200

3920 B.C.

2490 B.C.

1890 B.C.

1850 B.C.

25,850 B.C.

10,750 B.C.

22,250 B.C.

23,000 B.C.

4420 B.C.

23,450 B.C.

12,850 B.C.

20,050 B.C.

13,850 B.C.

A.D. 1770

320 B.C.

A.D. 1790

A.D. 1010

6110 B.C.

22,350 B.C. 


\begin{tabular}{|c|c|c|c|c|}
\hline $\begin{array}{l}\text { Laboratory } \\
\text { number }\end{array}$ & $\begin{array}{c}\text { Published } \\
\text { reference }\end{array}$ & $\begin{array}{c}\text { Original daie or } \\
\text { other value }\end{array}$ & $\begin{array}{l}\text { Corrected date } \\
\text { or other value }\end{array}$ & $\partial \mathrm{C}^{14}, \% 0$ \\
\hline-221 & $110: 114$ & $4040 \pm 180$ & & \\
\hline-222 & $110: 114$ & $9050 \pm 250$ & & \\
\hline-223 & $110: 113$ & $9900 \pm 600$ & & \\
\hline-224 & $110: 111$ & $24,100 \pm 400$ & & \\
\hline-225 & $110: 110$ & $30,200 \pm 3500$ & & \\
\hline-226 & $110: 115$ & $>30,000$ & & \\
\hline-227 & $110: 115$ & $>30,000$ & & \\
\hline-230 & $110: 110$ & $8650 \pm 200$ & & \\
\hline-231 & $110: 110$ & $>30,000$ & & \\
\hline-233 & $110: 112$ & $2530 \pm 130$ & & \\
\hline-235 & $110: 112$ & $3930 \pm 130$ & & \\
\hline-236 & $110: 112$ & $3000 \pm 190$ & & \\
\hline-237 & $110: 112$ & $3350 \pm 250$ & & \\
\hline-239 & $110: 112$ & $3900 \pm 600$ & & \\
\hline$-240 a$ & $110: 110$ & $26,600 \pm 1600$ & & \\
\hline-244 & $110: 114$ & $25,400 \pm 600$ & & \\
\hline-245 & $110: 114$ & $23,800 \pm 1100$ & & \\
\hline-246 & $110: 112$ & $1960 \pm 70$ & & \\
\hline-247 & $110: 115$ & $6460 \pm 130$ & & \\
\hline-248 & $110: 115$ & $7190 \pm 230$ & & \\
\hline-249 & $110: 111$ & $5470 \pm 450$ & & \\
\hline-252 & $110: 112$ & $>30,000$ & & \\
\hline .253 & $110: 111$ & $2830 \pm 120$ & & \\
\hline-255 & $110: 111$ & $21,200 \pm 950$ & & \\
\hline-256 & $151: 20$ & $575 \pm 135$ & & \\
\hline-257 & $151: 20$ & $680 \pm 360$ & & \\
\hline-258 & $151: 20$ & $2250 \pm 250$ & & \\
\hline-259 & $151: 20$ & $710 \pm 170$ & & \\
\hline-260 & $151: 20$ & modern & & \\
\hline-266 & $137: 203$ & $660 \pm 80$ & & \\
\hline-267 & $137: 200$ & $16,150 \pm 550$ & & \\
\hline-268 & $137: 201$ & $21,600 \pm 900$ & & \\
\hline-269 & $137: 201$ & $31,000 \pm 2500$ & & \\
\hline$-270: 1$ & $137: 197$ & $2 \pm 5.9$ & $-0.2 \pm 3.6 b$ & $+2.5 \pm 3.5 \mathrm{c}$ \\
\hline$-270: 2$ & $137: 197$ & $-8 \pm 3.0$ & $-11.9 \pm 3.1 \mathrm{~b}$ & $-9.1 \pm 2.9 \mathrm{c}$ \\
\hline$-270: 3$ & $137: 197$ & $-7 \pm 2.2$ & $-9.3 \pm 3.8 \mathrm{~b}$ & $-6.5 \pm 3.7 c$ \\
\hline$-270: 4$ & $137: 198$ & $-7 \pm 3.5$ & $-8.2 \pm 3.6 \mathrm{~b}$ & $-5.4 \pm 3.5 c$ \\
\hline$-270: 5$ & $137: 198$ & $-5 \pm 2.0$ & $-8.0 \pm 3.2 \mathrm{~b}$ & $-5.2 \pm 3.1 \mathrm{c}$ \\
\hline$-270: 6$ & $137: 198$ & $-12 \pm 1.9$ & $-9.0 \pm 3.3 \mathrm{~b}$ & $-6.2 \pm 3.2 \mathrm{c}$ \\
\hline$-270: 7$ & $137: 198$ & $-3 \pm 2.2$ & $-4.6 \pm 3.3 \mathrm{~b}$ & $-1.8 \pm 3.2 \mathrm{c}$ \\
\hline-272 & $137: 198$ & $\begin{array}{r}33,200+3800 \\
-2700\end{array}$ & & \\
\hline-273 & $137: 199$ & $>31,000$ & $>31,000 \mathrm{c}$ & \\
\hline-274 & $137: 198$ & $22,100 \pm 900$ & & \\
\hline .275 & $137: 210$ & $21,000 \pm 850$ & & \\
\hline-276 & $137: 206$ & $840 \pm 80$ & & \\
\hline-277 & $137: 203$ & $2250 \pm 420$ & & \\
\hline-278 & $137: 199$ & $6110 \pm 160$ & & \\
\hline-279 & $137: 199$ & $4870 \pm 150$ & & \\
\hline-280 & $137: 201$ & $2800 \pm 150$ & & \\
\hline .281 & $137: 201$ & $2240 \pm 150$ & & \\
\hline-282 & $137: 198$ & $\begin{array}{r}32,600+3200 \\
-2300\end{array}$ & & \\
\hline-283 & $137: 198$ & $30,600 \pm 3000$ & & \\
\hline-284 & $137: 199$ & $26,350 \pm 1100$ & & \\
\hline-285 & $137: 202$ & $25,400 \pm 1200$ & & \\
\hline-286 & $137: 203$ & $\begin{array}{r}34,000+3000 \\
-2000\end{array}$ & & \\
\hline-287 & $137: 203$ & $22,800 \pm 1000$ & & \\
\hline-288 & $137: 203$ & $29,500+2400$ & & \\
\hline
\end{tabular}

A.D./

B.C. date

2090 B.C.

7100 B.C.

7950 B.C.

22,150 B.C.

28,250 B.C.

6700 B.C.

580 B.C.

1980 B.C.

1050 B.C.

1400 B.C.

1950 B.C.

24,650 B.C.

23,450 B.C.

21,850 B.C.

10 B.C.

4510 B.C.

5240 B.C.

3520 B.C.

880 B.C.

19,250 B.C.

A.D. 1375

A.D. 1270

300 B.C.

A.D. 1210

A.D. 1290

14,200 B.C.

19,650 B.C.

29,050 B.C.

31,250 B.C.

20,150 B.C. 19,050 B.C. A.D. 1110 300 B.C.

4160 B.C. 2920 B.C. 850 B.C.

290 B.C.

30,650 B.C.

28,650 B.C. 24,400 B.C. 23,450 B.C.

32,050 B.C.

20,850 B.C.

27,550 B.C. 


\begin{tabular}{|c|c|c|c|c|}
\hline $\begin{array}{l}\text { Laboratory } \\
\text { number }\end{array}$ & $\begin{array}{l}\text { Published } \\
\text { reference }\end{array}$ & $\begin{array}{l}\text { Original date or } \\
\text { other value }\end{array}$ & $\begin{array}{l}\text { Corrected date } \\
\text { or other value }\end{array}$ & $\partial \mathrm{C}^{14}, \%$ \\
\hline-289 & $137: 203$ & $\begin{array}{l}31,200+2500 \\
-1900\end{array}$ & & \\
\hline-290 & $151: 20$ & modern & & \\
\hline-291 & $151: 20$ & $480 \pm 110$ & & \\
\hline $\begin{array}{l}-292 \\
.293\end{array}$ & $\begin{array}{l}137: 205 \\
137: 199\end{array}$ & $1800 \pm 120$ & & \\
\hline $\begin{array}{l}-293 \\
-294\end{array}$ & $137: 204$ & $\begin{array}{c}4450 \pm 140 \\
910 \pm 90\end{array}$ & & \\
\hline-295 & $137: 203$ & $750 \pm 120$ & & \\
\hline-296 & $137: 204$ & $900 \pm 100$ & & \\
\hline-297 & $137: 204$ & $5340 \pm 160$ & & \\
\hline-298 & $137: 204$ & $390 \pm 120$ & & \\
\hline-299 & $151: 22$ & $280 \pm 110$ & & \\
\hline-300 & $151: 22$ & $\mathrm{C}^{14}+5 \mathrm{I} \pm 11 \%$ & & $+51 \pm 11$ \\
\hline-301 & $137: 205$ & $1970 \pm 120$ & & \\
\hline-302 & $151: 20$ & $620 \pm 150$ & & \\
\hline-303 & $151: 20$ & modern & & \\
\hline-305 & $151: 22$ & modern & & \\
\hline-306 & $137: 204$ & $2570 \pm 110$ & & \\
\hline-309 & $137: 205$ & $900 \pm 120$ & & \\
\hline-310 & $137: 205$ & $1690 \pm 80$ & & \\
\hline-311 & $137: 201$ & $10,650 \pm 250$ & & \\
\hline-312 & $137: 200$ & $28,400 \pm 1800$ & & \\
\hline-314 & $137: 202$ & $27,900 \pm 1700$ & & \\
\hline-315 & $137: 205$ & $1850 \pm 90$ & & \\
\hline-316 & $137: 205$ & $1570 \pm 100$ & & \\
\hline-317 & $137: 205$ & $1460 \pm 100$ & & \\
\hline-318 & $137: 201$ & $16,500 \pm 400$ & & \\
\hline-319 & $137: 198$ & $19,400 \pm 700$ & & \\
\hline-320 & $137: 200$ & $>32,000$ & & \\
\hline-321 & $137: 200$ & $23,700 \pm 1100$ & & \\
\hline-322 & $137: 200$ & $16,800 \pm 500$ & & \\
\hline-324 & $137: 205$ & $1530 \pm 80$ & & \\
\hline-325 & $151: 20$ & $1160 \pm 100$ & & \\
\hline-329 & $137: 199$ & $14,500 \pm 600$ & & \\
\hline-330 & $137: 203$ & $690 \pm 90$ & & \\
\hline-334 & $137: 206$ & $11,600 \pm 400$ & & \\
\hline-335 & $137: 206$ & $18,800 \pm 800$ & & \\
\hline-336 & $137: 206$ & $1470 \pm 120$ & & \\
\hline-337 & $137: 206$ & $140 \pm 100$ & & \\
\hline-344 & $137: 202$ & $20,800 \pm 1000$ & & \\
\hline$-351 \mathrm{a}$ & $151: 13$ & $1330 \pm 90$ & & \\
\hline$-351 b$ & $151: 14$ & $1350 \pm 100$ & & \\
\hline-352 & $151: 14$ & $2420 \pm 150$ & & \\
\hline-353 & $151: 14$ & $1500 \pm 160$ & & \\
\hline-362 & $151: 12$ & $2260 \pm 90$ & & \\
\hline-363 & $151: 12$ & $1610 \pm 80$ & & \\
\hline-364 & $151: 21$ & modern & & \\
\hline-365 & $151: 21$ & $350 \pm 110$ & & \\
\hline-366 & $151: 21$ & modern & & \\
\hline-367 & $151: 22$ & modern & & \\
\hline-368 & $151: 22$ & modern & & \\
\hline-369 & $151: 22$ & $350 \pm 100$ & & \\
\hline-370 & $151: 16$ & $1210 \pm 30$ & & \\
\hline-371 & $151: 16$ & $310 \pm 100$ & & \\
\hline-372 & $151: 16$ & $1640 \pm 120$ & & \\
\hline-373 & $151: 16$ & $1870 \pm 140$ & & \\
\hline-374 & $151: 17$ & $2580 \pm 150$ & & \\
\hline-375 & $151: 17$ & $2760 \pm 160$ & $2670 \pm 160 \mathrm{c}$ & \\
\hline-376 & $151: 17$ & $2870 \pm 130$ & & \\
\hline-378 & $151: 17$ & modern $<250$ & & \\
\hline$-379 \mathrm{a}$ & $151: 16$ & $4730 \pm 90$ & & \\
\hline
\end{tabular}

A.D./

B.C. date

29,250 B.C.

A.D. 1470

A.D. 150

2500 B.C.

A.D. 1040

A.0. 1200

A.D. 1050

3390 B.C.

A.D. 1560

A.D. 1670

20 B.C.

A.D. 1330

620 B.C.

A.D. 1050

A.D. 260

8700 B.C.

26,450 B.C.

25,950 B.C.

A.D. 100

A.D. 380

A.D. 490

14,550 B.C.

17,450 B.C.

21,750 B.C. 14,850 B.C.

A.D. 420

A.D. 790

12,550 B.C.

A.D. 1260

9650 B.C.

16,850 B.C.

A.D. 480

A.D. 1810

18,850 B.C.

A.D. 620

A.D. 600

470 B.C.

A.D. 450

310 B.C.

A.D. 340

A.D. 1600

A.D. 1600

A.D. 740

A.D. 1640

A.D. 310

A.D. 80

630 B.C.

720 B.C.

920 B.C.

2780 B.C. 


\begin{tabular}{|c|c|c|c|c|c|}
\hline $\begin{array}{l}\text { Laboratory } \\
\text { number }\end{array}$ & $\begin{array}{l}\text { Published } \\
\text { reference }\end{array}$ & $\begin{array}{l}\text { Original date or } \\
\text { other value }\end{array}$ & $\begin{array}{l}\text { Corrected date } \\
\text { or other value }\end{array}$ & $\partial \mathrm{C}^{14}, \%$ & $\begin{array}{c}\text { A.D./ } \\
\text { B.C. date }\end{array}$ \\
\hline$-379 b$ & $151: 16$ & $4760 \pm 90$ & & & 2810 B.C. \\
\hline-381 & $151: 11$ & $25,900 \pm 1000$ & & & 23,950 B.C. \\
\hline-382 & $151: 14$ & $>35,000$ & & & \\
\hline-383 & $151: 11$ & $17,200 \pm 4.00$ & & & 15,250 B.C. \\
\hline-384 & $151: 14$ & $>33,000$ & & & \\
\hline-385 & $151: 14$ & $12,000 \pm 250$ & & & 10,050 B.C. \\
\hline-386 & $151: 11$ & $28,400 \pm 1700$ & & & 26,450 B.C. \\
\hline-387 & $151: 14$ & $17,300 \pm 300$ & & & 15,350 B.C. \\
\hline-388 & $151: 11$ & $>34,000$ & & & \\
\hline-389 & $151: 15$ & $1500 \pm 100$ & & & A.D. 450 \\
\hline-390 & $151: 15$ & $1380 \pm 90$ & & & A.D. 570 \\
\hline-391 & $151: 13$ & $3800 \pm 130$ & & & 1850 B.C. \\
\hline$-393 b$ & $151: 18$ & $2500 \pm 400$ & & & 550 B.C. \\
\hline$-394 a$ & $151: 18$ & $3880 \pm 150$ & & & 1930 B.C. \\
\hline$-394 \mathbf{b}$ & $151: 18$ & $3000 \pm 120$ & & & 1050 B.C. \\
\hline-397 & $151: 19$ & $8250 \pm 60$ & & & 6300 B.C. \\
\hline-398 & $151: 19$ & $760 \pm 50$ & & & A.D. 1190 \\
\hline-405 & $151: 15$ & $930 \pm 150$ & & & A.D. 1020 \\
\hline-406 & $151: 15$ & $3500 \pm 100$ & & & 1550 B.C. \\
\hline-407 & $151: 15$ & $3600 \pm 190$ & & & 1650 B.C. \\
\hline-408 & $151: 15$ & $1400 \pm 250$ & & & A.D. 550 \\
\hline-409 & $151: 12$ & $19,600 \pm 600$ & & & 17,650 B.C. \\
\hline-4.11 & $151: 12$ & $4790 \pm-110$ & & & 2840B.C. \\
\hline-412 & $151: 13$ & $4950 \pm 150$ & & & 3000 B.C. \\
\hline-414 & $151: 11$ & $30,000 \pm 1700$ & & & 28,050 B.C. \\
\hline$-4,15$ & $151: 12$ & 33,200 & & & 31,250 B.C. \\
\hline & & $\begin{array}{r}+4100 \\
-2700\end{array}$ & & & \\
\hline-422 & $151: 19$ & $820 \pm 90$ & & & A.D. 1130 \\
\hline .423 & $151: 19$ & $6350 \pm 100$ & & & 4400 B.C. \\
\hline-424 & $151: 19$ & $320 \pm 90$ & & & A.D. 1630 \\
\hline-425 & $151: 19$ & $380 \pm 90$ & & & A.D. 1570 \\
\hline-426 & $151: 19$ & $1020 \pm 40$ & & & A.D. 930 \\
\hline-427 & $151: 19$ & $7450 \pm 270$ & & & 5500 B.C. \\
\hline-428 & $151: 19$ & $7030 \pm 40$ & & & 5080 B.C. \\
\hline-429 & $151: 19$ & $8600 \pm 300$ & & & 6650 B.C. \\
\hline-440 & $151: 22$ & $5070 \pm 140$ & & & 3120 B.C. \\
\hline-442 & $151: 13$ & $690 \pm 90$ & & & A.D. 1260 \\
\hline-451 & $151: 10$ & $4360 \pm 140$ & & & 2410 B.C. \\
\hline-452 & $151: 10$ & $4370 \pm 130$ & & & 2420 B.C. \\
\hline-453 & $151: 10$ & $2740 \pm 100$ & & & 780 B.C. \\
\hline-454 & $151: 11$ & $6630 \pm 150$ & & & 4680 B.C. \\
\hline-455 & $151: 11$ & $27,200 \pm 1200$ & & & 25,250 B.C. \\
\hline-456 & $151: 12$ & $20,200 \pm 800$ & & & 18,250 B.C. \\
\hline-457 & $151: 12$ & $17,900 \pm 450$ & & & 15,950 B.C. \\
\hline-458 & $151: 13$ & $1580 \pm 100$ & & & A.D. 370 \\
\hline-459 & $151: 13$ & $1600 \pm 100$ & & & A.D. 350 \\
\hline-460 & $151: 15$ & $10,400 \pm 220$ & & & 8450 B.C. \\
\hline$-462^{1}$ & $151: 18$ & modern & pub. as GaK-482 & & \\
\hline-463 & $151: 17$ & $1090 \pm 60$ & & & A.D. 860 \\
\hline-464 & $151: 17$ & $1230 \pm 50$ & & & A.D. 720 \\
\hline-476 & $151: 14$ & $21,230 \pm 720$ & & & 19,280 B.C. \\
\hline-481 & $151: 18$ & $1580 \pm 130$ & & & A.D. 370 \\
\hline-482 & $151: 18$ & $7450 \pm 180$ & & & 5500 B.C. \\
\hline$-482^{1}$ & $151 \cdot 18$ & & & & \\
\hline-483 & & modern & & & \\
\hline
\end{tabular}

${ }^{1}$ Original lab. nos. of GaK-163 (Nagai, Osaka, > 30,000), GaK 163 (Daisen, $3380 \pm 60$ ) and GaK-482 (Curracurrang 15M, a, Modern) are changed to GaK-163b, GaK-163a, and GaK-462 respectively. 